\title{
Acute Kidney Injury in Critically Injured Combat Veterans: A Retrospective Cohort Study
}

Ian J. Stewart MD ${ }^{1,2}$, Jonathan A. Sosnov MD ${ }^{2,3}$, Jeffery T. Howard $\mathrm{PhD}^{4}$, and Kevin K. Chung $\mathrm{MD}^{2,4}$

${ }^{1}$ David Grant Medical Center, Clinical Investigation Facility, ${ }^{2}$ Uniformed Services University of the Health Sciences, ${ }^{3}$ San Antonio Military Medical Center, ${ }^{4}$ U. S. Army

Institute of Surgical Research

\section{Corresponding author}

Maj Ian J. Stewart MD, USAF, MC

David Grant Medical Center

Clinical Investigation Facility

101 Bodin Circle

Travis AFB, CA 94535

Phone: 707-423-7264, Fax: 707-423-7267

Email: ian.stewart@us.af.mil

Short title: AKI in critically injured combat veterans 
Background: Acute kidney injury (AKI) has been associated with mortality after traumatic injury. However, there is a paucity of data in military service members with injuries suffered in combat. We sought to identify risk factors for AKI after combat trauma and to evaluate if AKI is a predictor of mortality.

Study Design: Retrospective, observational study.

Settings \& Participants: US service members who were critically wounded in Iraq or Afghanistan from 1 Feb 2002 to 1 Feb 2011 who survived until evacuation to Landstuhl Regional Medical Center, Germany.

Predictors: Demographic variables, vital signs, injury severity score, presence of burn injury, and mechanism of injury as defined at the time of initial injury, as well as the presence of AKI ascertained within the first 7 days using the KDIGO (Kidney Disease: Improving Global Outcomes) serum creatinine criteria.

Outcomes: Logistic regression models were used to identify risk factors for both AKI and death.

Results: Of 6,011 records, 3,807 were included for analysis after excluding patients with missing data. AKI occurred in 474 patients (12.5\%) and 112 (2.9\%) died. More patients with versus without AKI died $(\mathrm{n}=62[13.1 \%]$ versus $\mathrm{n}=50$ [10.5\%]; $\mathrm{P}<0.001)$. After adjustment, AKI was a predictor of mortality (OR, 5.14; 95\% CI, 3.33-7.93; p <0.001). Predictors of AKI were age, African-American race, injury severity score, amputations, burns, and presenting vital signs.

Limitations: AKI diagnoses limited to creatinine-based definitions.

Conclusions: AKI predicted mortality in combat veterans injured in the wars in Iraq and Afghanistan.

Keywords: Acute kidney injury (AKI), trauma, combat veteran, mortality, death, risk factors, combat, military service, war casualty, military personnel, serum creatinine, KDIGO AKI criteria, burn injury 
Acute kidney injury (AKI) has been studied in a wide range of settings and patient populations including those with severe trauma. ${ }^{1-6}$ However, to our knowledge the overall rate of AKI among those severely injured during the course of combat operations in Iraq and Afghanistan has not been systematically examined in a large cohort.

While data in the combat injured population are limited to those with burn injury ${ }^{7}$, a small cohort of critically injured combat casualties ${ }^{8}$ and those who required renal replacement therapy (RRT) ${ }^{9}$, there is a body of evidence from the civilian trauma population. The rate of AKI reported in civilian trauma ranges from $6 \%$ to $50 \%$ and is associated with a $14.9 \%-57 \%$ mortality rate. This wide range in estimates represents different patient populations, different definitions of AKI, and different time courses examined.

Three of these studies have used the RIFLE (risk, injury, failure, loss, end-stage renal disease $)^{10}$ criteria to classify AKI. The first was a large cohort of critically injured trauma patients $(\mathrm{N}=9,449)$. After admission, AKI was assessed for the first 24 hours and occurred in $18.1 \% .^{1}$ It was associated with a $16.7 \%$ mortality rate compared with $7.8 \%$ for those without AKI. The second study found that AKI occurred in $26 \%$ of 982 patients with severe blunt trauma over the first 28 days after injury. ${ }^{2}$ Patients without AKI had a mortality rate of $5 \%$ compared to $32 \%$ for those with AKI. The third study, examined 666 patients admitted to a trauma intensive care unit (ICU). AKI occurred in $15 \%$ and was associated with a mortality rate of $57 \%{ }^{6}$; the high mortality rate in this study is an outlier, possibly because over half of the AKI in this study was RIFLE class failure.

Two studies have used the AKI Network (AKIN) criteria ${ }^{11}$ to define AKI in ICU 
trauma cohorts. In the first $(\mathrm{N}=400)$, AKI occurred in $36.8 \%$ of patients within 5 days of injury. ${ }^{5}$ It was associated with a mortality rate of $14.9 \%$ compared with $3.8 \%$ for those without AKI. In the second study of 901 patients, early AKI (defined as within 72 hours of admission) occurred in $6 \%$ of patients. ${ }^{4}$ Patients with AKI were more likely to die (29.6\%) compared to patients without AKI (7.9\%).

One recent study utilized the KDIGO (Kidney Disease: Improving Global Outcomes $)^{12}$ criteria to define AKI in 412 patients with trauma admitted to an ICU. ${ }^{3}$ These authors found that AKI occurred in $24.9 \%$ of patients within the first 7 days after injury and was associated with a higher risk of death at both 30 days (17.5\% vs $5.8 \%)$ and 1 year after injury (26.2\% vs $7.1 \%)$.

Given the strong association between AKI and mortality in the civilian trauma population, and the paucity of data in combat veterans, we sought to describe the epidemiology, outcomes and predictors for AKI in a large cohort of critically injured combat casualties.

\section{Methods}

This study was conducted under a protocol reviewed and approved by the US Army Medical Research and Materiel Command Institutional Review Board (IRB) and in accordance with the approved protocol (log number M-10223). The IRB waived the need for informed consent.

Data were collected from a variety of Department of Defense databases, including the Department of Defense Trauma Registry, the Armed Forces Medical Examiner System and data systems of the Defense Health Agency. Patient selection and data sources have

been previously described. ${ }^{13}$ Briefly, US military personnel who were injured in combat 
from $1 \mathrm{Feb} 2002$ to $1 \mathrm{Feb} 2011$, required ICU level care and survived to be evacuated out of Iraq or Afghanistan were included in the cohort. Data collected for this study included demographic variables (age, gender, and race), mechanism of injury, presenting vital signs (heart rate, temperature, and mean arterial blood pressure [MAP]), injury severity score (ISS) ${ }^{14}$ utilizing the 2005 abbreviated injury scale update ${ }^{15}$, creatinine and 90 day mortality (obtained from the Armed Forces Medical Examiner System).

To be included for analysis, a patient must have had at least one creatinine value measured in the first 7 days after injury. Subjects were excluded if data were missing for one of the pre-specified covariates (with the exception of race). Excluded individuals were examined and compared with the full-case sample to determine differences across demographic and injury data where available. Sensitivity analyses were performed to determine the extent to which estimates based on the full-case sample may differ from the total sample. For individuals missing demographic or injury data, we performed sensitivity analyses by imputing the mean value and by using multiple regression techniques to impute missing values based on the combination of other covariates. For individuals missing creatinine data, we estimated models assuming all missing cases had AKI (following the observed distribution by KDIGO stage), and assuming all missing cases did not have AKI. This established upper and lower bounds for our estimates based on variability attributable to missing data.

The KDIGO criteria $^{12}$ served as the basis for determining AKI. We did not have data on urine output; therefore only changes in creatinine were used to determine AKI stage. When available, a known creatinine value (drawn 7-365 days prior to the date of injury) was used as the baseline. If the patient did not have a known pre-injury creatinine, we 
back-calculated one using the CKD-EPI (Chronic Kidney Disease Epidemiology Collaboration) creatinine equation ${ }^{16}$, assuming an eGFR of $100 \mathrm{ml} / \mathrm{min} / 1.73 \mathrm{~m}^{2}$. If a back-calculated creatinine was used, and race was unknown, it was assumed to be nonAfrican American. Assessment for AKI was determined for 7 days after injury. The need for RRT was defined by the International Classification of Diseases, $9^{\text {th }}$ edition (ICD-9) codes V568 and V560.

The ISS is a validated anatomic based scoring system used to quantify the severity of injury. The score ranges from 1 to 75 , increasing with severity. ${ }^{14}$ This method has been used to quantify severity of injury in multiple studies that use data from the Department of Defense Trauma Registry ${ }^{17,18}$ and studies that have examined AKI in the setting of trauma. ${ }^{2-6}$ We modeled a variety of functional forms for ISS including continuous, quadratic, log, exponential and categorical and compared them using Akaike information criterion. None of the other methods were superior to considering ISS as a continuous variable. Given our observation that outcomes appear to be worse for patients at extremes of MAP, this variable was divided into three categories. We chose cutoffs reflecting a level that might require intervention in the low group $\left(<65 \mathrm{mmHg}^{19}\right)$ and a value representing hypertension $\left(>106 \mathrm{mmHg}^{20}\right)$ in the high group.

Standard descriptive statistics were used to compare the group of patients who developed AKI with those who did not. While race was assumed to be non-African American for the purposes of back-calculation, race missing was considered as a separate category in subsequent analyses. Two different univariate and multivariable analyses were performed. The first was a logistic regression model examining the effect of baseline injury characteristics on the subsequent development of AKI. The second 
analysis examined 90 day mortality, with baseline injury characteristics and AKI as independent variables. All variables were included in the multivariable analyses. Statistics were performed using SAS version 9.2 (SAS Institute Inc, Cary, NC).

\section{Results}

Of the 6,011 records identified, 3,807 patients were included for analysis. Reasons for exclusion were no recorded creatinine within 7 days after injury ( $\mathrm{n}=1159)$, no recorded heart rate $(\mathrm{n}=301)$, no recorded MAP $(\mathrm{n}=89)$ and no recorded temperature $(\mathrm{n}=655)$. Only $7.2 \%(\mathrm{n}=276)$ of patients had a known baseline for the purposes of AKI staging. Baseline characteristics are presented in Table 1. There were 474 (12.5\%) of patients in whom AKI occurred. More patients with AKI died compared to patients without AKI (13.1\% vs 10.5\%, respectively; $\mathrm{p}<0.001)$. Patients with AKI were more likely to have burn injury compared to patients without AKI (28.1\% vs $17.6 \%$, respectively, $\mathrm{p}<0.001$ ). The median ISS for patients with AKI was 26 (interquartile range [IQR], 17-36), which was higher than for patients without AKI (14; IQR, 9-22; $\mathrm{p}<0.001)$. Patients with AKI were slightly, but significantly, older than patients without AKI (mean age, $27 \pm 7$ [standard deviation] vs $25 \pm 6$ years; $\mathrm{p}=0.002$ ). Amputations were more common in patients with AKI (28.3\% vs $17.7 \%$; $\mathrm{p}<0.001)$. Differences in both heart rate and MAP category were also observed.

When stratified by AKI stage, $9.8 \%, 1.6 \%$ and $1.1 \%$ of patients had KDIGO stages 1 through 3, respectively. As shown in Figure 1, mortality increased across AKI stage with rates of 1.5\%, 7.0\%, 23.7\% and 53.7\%, for no AKI and KDIGO stages 1, 2, and 3, respectively $(\mathrm{p}<0.001)$. Fourteen patients with AKI required RRT at a median of 15.5 (IQR, 8.8-20.3) days after injury; 3 of these patients required dialysis within the first 7 
days after injury. Five patients (36\%) who required RRT died within 90 days. Figure 2 demonstrates the percentage of AKI patients who were diagnosed with AKI on any given hospital day.

The results of the models for the outcome of AKI are presented in Table 2. On multivariable analysis, age (odds ratio [OR] per 1 year older, 1.03; 95\% CI, 1.02-1.05; $\mathrm{p}<0.001$ ), ISS (OR per 1-point greater, 1.05; 95\% CI, 1.05-1.06; $\mathrm{p}<0.001$ ), burn injury (OR, 1.77; 95\% CI, 1.37-2.27; $\mathrm{p}<0.001)$, heart rate (OR per 1-beat/min greater, 1.01; 95\% CI, 1.00-1.01; $\mathrm{p}=0.001)$, African American race (OR, 1.56; 95\% CI, 1.03-2.36; $\mathrm{p}=0.04)$, amputation $(\mathrm{OR}, 1.46 ; 95 \% \mathrm{CI}, 1.14-1.88 ; \mathrm{p}=0.003)$, gunshot wound injury (vs explosive injury; OR, 1.53 ; 95\% CI, 1.16-2.03; $\mathrm{p}=0.003)$, temperature $\left(\mathrm{OR}\right.$ per $1^{\circ} \mathrm{F}$ greater, 1.08; 95\% CI, 1.05-1.84; $\mathrm{p}=0.01$ ), and low and high MAP (ORs of 1.60 [95\% CI, 1.20-2.14; $\mathrm{p}=0.002]$ ) and 1.39 [95\% CI, 1.05-1.84; $\mathrm{p}=0.02$ ] compared to normal MAP) were predictors for the subsequent development of AKI.

The results of the models for 90 day mortality are presented in Table 3. In the multivariable model, ISS (OR per 1-point greater, 1.07; 95\% CI, 1.06-1.08; p<0.001), heart rate (OR per 1-beat/min greater, 0.99; 95\% CI, 0.98-1.00; $\mathrm{p}=0.03)$, temperature (OR per $1^{\circ} \mathrm{F}$ greater, $\left.0.90 ; 95 \% \mathrm{CI}, 0.82-0.99 ; \mathrm{p}=0.04\right)$, and low and high MAP (ORs of 2.33 [95\% CI, 1.36-4.01; $\mathrm{p}=0.002]$ and 1.98 [95\% CI, 1.13-3.47; $\mathrm{p}=0.02]$ compared to normal MAP) were predictors of death. When stratified by KDIGO stage, the odds of death increased in a dose-dependent manner compared to patients with no AKI in the multivariable model (Table 3). When considered as a dichotomous variable, the presence of AKI increased the odds of death by over five-fold (OR, 5.14; 95\% CI, 3.337.93; $\mathrm{p}<0.001$ ) compared to patients without AKI after adjustment. While burn injury 
predicted mortality on univariate analysis, this difference did not persist after adjustment. While increased heart rate predicted mortality in the univariate model, it was associated with less mortality in the multivariable model. Further analysis with the inclusion of an interaction term between MAP categories and heart rate suggested that this may be due to different implications of heart rate across different MAP categories.

Examination of excluded individuals $(n=2204)$ demonstrated that they were less likely to have a burn injury (13\% vs. 19\%), less likely to have normal MAP (63\% vs. 76\%), had lower mean heart rates (92 vs. 97 beats/min), and had less severe injuries (mean ISS, 12 vs. 18) compared with included cases. Sensitivity analyses for the AKI model produced similar multivariable results with the exception of burn injury. Under the assumption that all patients missing creatinine values had AKI, burn injury was no longer statistically significant $(\mathrm{p}=0.2)$. All other results for AKI were similar. For mortality and KDIGO stages 1,2, and 3, minimum OR estimates were 2.75, 5.23 and 22.07, respectively, and maximum OR estimates were 3.02, 6.01 and 27.70, respectively. This suggests that the missing data do not materially change the results.

\section{Discussion}

We found that AKI occurred in $12.5 \%$ of our large cohort of critically injured combat casualties. Predictors for the development of AKI were age, African American race, ISS, amputation, presence of burn injury, gunshot wound, heart rate, temperature, as well as both low and high MAP. After adjustment, AKI was strongly associated with mortality, imparting an over 5 -fold increase in the risk of death. When stratified by stage, the odds of death increased in a dose-response fashion.

Our group has previously reported two studies; one examining the rate of AKI in 
combat related burn and the other describing early AKI in combat related trauma. ${ }^{7,8}$ While both studies represented progress in the field of AKI as it relates to combat injury, they both had limitations. In the first study of 692 casualties with burn injury ${ }^{7}, \mathrm{AKI}$ occurred in $29.9 \%$ of patients (by the AKIN criteria ${ }^{11}$ ) and was predictive of mortality. This work was limited to patients with burn injury, did not have creatinine data prior to admission to a hospital in the United States and did not have data on baseline injury characteristics. The rate of AKI seen in these burn injured patients was higher than what we observed in the present study. This difference is likely explained by the higher risk of AKI in burn injury (Table 2). In the other study ${ }^{8}$, AKI was found to occur in $34.3 \%$ (by KDIGO criteria $^{12}$ ) of select combat casualties enrolled into one of two prospective cohorts. While the mortality in the AKI group was higher than that in the group without AKI (21.7\% vs $2.3 \%$, respectively), the small number of patients in the cohort $(\mathrm{N}=134)$ and small number of deaths $(\mathrm{n}=12)$ limited the ability to perform a multivariable analysis. The rate of $34.3 \%$ in this small cohort was higher than we observed in the present study. This difference is likely attributed to more severe injuries, as evidenced by higher median ISS (21 vs 14) and higher mortality (9.0\% vs $2.9 \%$ ), compared with the present work. Consistent between all three studies however is timing of AKI, with the majority of cases occurring early after injury.

Data on 51 combat casualties that required RRT were published by Bolanos and colleagues. ${ }^{9}$ These investigators found that mortality was $21.6 \%$ at 60 days. This is seemingly lower than the mortality we observed in patients with KDIGO stage $3(53.7 \%)$ and those who required RRT (35.7\%). The lower mortality in the study by Bolanos et al may be due to different inclusion criteria. To be included in their study, casualties must 
have survived to be evacuated back to the United States, which could have selected a less severely injured cohort. It should also be noted that their method of identifying dialysis patients (review of medical records) was likely more sensitive than our method (ICD-9 codes from an administrative database).

A well-known complication of burn injury, AKI is associated with high mortality rates $^{21-23}$, however there is a paucity of data comparing AKI rates in burn injury with those in other forms of trauma. Our work indicates that patients with burn injury are at an increased risk after adjustment. We also observed a strong correlation of AKI with ISS, which was consistent with our prior study. ${ }^{8}$ While a similar association was observed in one study ${ }^{3}$, most work in civilian populations has not observed ISS to be predictive of AKI., 4-6 Military personnel are young (mean age in this study, 26 years) and must meet physical standards to deploy to a combat zone. It is therefore possible that these differences are due to baseline patient characteristics, as civilian cohorts are older and have more co-morbid conditions. While age has been found to be predictive of AKI in trauma patients in multiple studies ${ }^{3,5-6}$, heart rate and MAP are less well studied and have led to conflicting results. ${ }^{2,3}$ Our data provide evidence for the intuitive notion that presenting hemodynamics affect subsequent AKI risk.

Another predictor of AKI in our model was amputation. Previous work in combat casualties has demonstrated that post-injury creatinine levels are lower in patients with amputations $^{24,25}$, which is likely a result of decreased creatinine production. While this might be expected to decrease the risk of AKI, we observed the opposite to be true. There are two possible explanations for our finding that amputation was a predictor for AKI. The first is that amputation might be another marker of injury severity. The second 
is that amputation is a marker for rhabdomyolysis, which increases AKI risk. We have previously shown that injuries to the extremities increase the risk of rhabdomyolysis, which in turn increases the subsequent risk of AKI. ${ }^{26}$

In our study, African Americans had a disproportionately high risk of AKI compared with other races. While this has been observed in one previous study in the trauma population, ${ }^{5}$ these results were not duplicated in another study ${ }^{2}$. There are three plausible explanations for racial disparity in AKI. Firstly, it is possible that this represents an increased risk of AKI driven by genetic factors. Secondly, socioeconomic factors, such as disparities in income or access to care may drive the association. Lastly, this might be due to increased creatinine production in African Americans compared with other racial backgrounds. It is important to note that in our study, socioeconomic factors are controlled for as all subjects had equal access to health care by virtue of being active-duty US service members. Further work on racial differences in AKI rates is clearly warranted to elucidate the increased risk of AKI in African Americans.

The mortality of patients with AKI in this study (13.1\%) is lower than what we have observed in our prior cohorts of combat injured patients $(20.3 \%-24.2 \%)^{7,8}$ and what has been seen in civilian populations that used one of the standardized AKI diagnostic criteria $(14.9 \%-57 \%) .{ }^{1-6}$ However, in the multivariable model for mortality, AKI was a predictor of death even after adjustment for other covariates including ISS, heart rate, MAP and temperature. Furthermore, when stratified by KDIGO stage of AKI, the odds of death increased in a dose-response fashion. While a cause and effect relationship cannot be established by a retrospective study, our findings demonstrate that AKI is a predictor of mortality even in a young cohort without the multiple confounding variables and 
comorbidities present in older patient populations.

Our findings are clinically relevant to those caring for critically injured combat casualties. The development and severity of AKI are predictive of mortality, which is useful for determining prognosis. Also, with knowledge of the risk factors for AKI after combat injury, clinicians can proactively avoid nephrotoxic exposures (e.g. contrast, aminoglycosides and nonsteroidal anti-inflammatory drugs) in high risk patients.

Our work has several limitations. The first one is the amount of missing data, which resulted in the exclusion of about a third of the initial study population. However, this data was collected in an active combat zone and represents the only data of its kind. Furthermore, sensitivity analyses of missing cases did not materially change the results. Secondly, without data on urine output, we could not use the full KDIGO criteria to define AKI. Recent evidence indicates that the risk of death is at its peak when both creatinine and urine output are considered. ${ }^{27}$ Had this information been available, we could have further refined our models. We also only had access to baseline creatinine in a minority of patients. However, back calculation appears to be a reasonable method in cohorts of patients with low prevalence of chronic kidney disease, such as ours. ${ }^{28}$ Thirdly, while we were able to adjust for demographic variables and baseline injury characteristics, we were unable to adjust for other in-hospital factors, such as acute respiratory distress syndrome, fungal infections and sepsis) that might affect outcomes. Lastly, the findings from our study in a young, predominantly male population with combat casualties may not be generalizable to other populations.

In conclusion, AKI was strongly predictive of mortality in combat veterans injured in Iraq and Afghanistan. Age, African-American race, ISS, amputation, presence of burn 
injury, gunshot wound mechanism of injury, heart rate, temperature, as well as both high and low MAP were predictors of AKI in this population. While these results underscore the possible role of AKI in the pathway from injury to death in combat casualties, retrospective studies such as this cannot infer causation. Therefore, the role of AKI as a possible factor in mortality after trauma generally, and combat trauma specifically, deserves further study.

\section{Acknowledgements}

The authors acknowledge the Department of Defense Trauma Registry, the Armed Forces Medical Examiner System and the Defense Health Agency for providing data for the study. The opinions or assertions contained herein are the private views of the authors and are not to be construed as official or as reflecting the views of the Department of the Air Force, the Department of the Army, or the Department of Defense.

Support: This project was supported with intramural funds from the US Army Institute of Surgical Research and by a postdoctoral fellowship provided by the Oak Ridge Institute of Science and Education (Dr Howard). The funders did not have a role in study design, data collection, data analysis, data interpretation, manuscript preparation, or the decision to submit for publication.

Financial Disclosure: The authors declare that they have no other relevant financial interests.

Contributions: Study conception: IJS; study design: IJS, KKC; data collection: IJS, KKC; data analysis/interpretation: JAS, JTH, KKC. Each author contributed important intellectual content during manuscript drafting or revision and accepts accountability for the overall work by ensuring that questions pertaining to the accuracy or integrity of any 
portion of the manuscript are appropriately investigated and resolved. IJS takes

responsibility that this study has been reported honestly, accurately, and transparently;

that no important aspects of the study have been omitted; and that any discrepancies from

the study as planned have been explained.

Peer Review: Evaluated by 3 external peer reviewers, a Statistical Editor, a Co-

Editor, and the Editor-in-Chief.

\section{$\underline{\text { References }}$}

1. Bagshaw S, George C, Gibney R, Bellomo R. A multi-center evaluation of early acute kidney injury in critically ill trauma patients. Ren Fail. 2008;30(6):581-589.

2. Bihorac A, Delano M, Schold J, et al. Incidence, clinical predictors, genomics, and outcome of acute kidney injury among trauma patients. Ann Surg. 2010;252(1):158-165.

3. Eriksson M, Brattstrom O, Martensson J, Larsson E, A O. Acute kidney injury following severe trauma: Risk factors and long-term outcome. J Trauma Acute Care Surg. 2015;79(3):407-412.

4. Podoll A, Kozar R, Holcomb J, Finkel K. Incidence and outcome of early acute kidney injury in critically-ill trauma patients. PloS One. 2013;8(10):e77376.

5. Shashaty M, Meyer N, Localio A, et al. African American race, obesity, and blood product transfusion are risk factors for acute kidney injury in critically ill trauma patients. J Crit Care. 2012;27(5):496-504.

6. Skinner D, Hardcastle T, Rodseth R, Muckhart D. The incidence and outcomes of acute kidney injury amongst patients admitted to a level I trauma unit. Injury. 2014;45(1):259-264.

7. Stewart I, Tilley M, Cotant C, et al. Association of AKI with Adverse Outcomes in Burned Military Casualties. Clin J Am Soc Nephrol. 2012;7(2):199-206.

8. Heegard K, Stewart I, Cap A, et al. Early acute kidney injury in military casualties. J Trauma Acute Care Surg. 2015;78(5):988-993.

9. Bolanos J, Yuan C, Little D, et al. Outcomes After Post-Traumatic AKI Requiring RRT in United States Military Service Members. Clin J Am Soc Nephrol. 2015;10(10):1732-1739.

10. Bellomo R, Ronco C, Kellum J, Mehta R, Palevsky P. Acute renal failure definition, outcome measures, animal models, fluid therapy and information technology needs: the Second International Consensus Conference of the Acute Dialysis Quality Initiative (ADQI) Group. Crit Care. 2004;8(4):R204-212.

11. Mehta R, Kellum J, Shah S, et al. Acute Kidney Injury Network: report of an initiative to improve outcomes in acute kidney injury. Crit Care. 2007;11(2):R31.

12. Kidney Disease: Improving Global Outcomes (KDIGO) Acute Kidney Injury Work Group. KDIGO Clinical Practice Guideline for Acute Kidney Injury. Kidney Inter. 2012;Suppl 2:1-138. 
13. Stewart I, Fang R, Cannon J, et al. Derivation of candidates for the combat casualty critical care (C4) database. Mil Med. 2014;179(4):370-374.

14. Baker S, O'Neill B, Haddon W, Long W. The Injury Severity Score: a method for describing patients with multiple injuries and evaluating emergency care. $J$ Trauma. 1974;14(3):187-196.

15. Gennarelli T, Wodzin E. AIS 2005: a contemporary injury scale. Injury. 2006;37(12):1083-1091.

16. Levey A, Stevens L, Schmid C, et al. A new equation to estimate glomerular filtration rate. Ann Intern Med. 2009;150(9):604-612.

17. Kotwal R, Howard J, Orman J, et al. The Effect of a Golden Hour Policy on the Morbidity and Mortality of Combat Casualties. JAMA Surg. 2016;151(1):15-24.

18. Stewart I, Sosnov J, Howard J, et al. Retrospective Analysis of Long-Term Outcomes After Combat Injury: A Hidden Cost of War. Circulation. 2015;132(22):2126-2133.

19. Dellinger R, Levy M, Rhodes A, et al. Surviving sepsis campaign: international guidelines for management of severe sepsis and septic shock: 2012. Intensive Care Med. 2013;39(2):165-228.

20. James P, Oparil S, Carter B, et al. 2014 evidence-based guideline for the management of high blood pressure in adults: report from the panel members appointed to the Eighth Joint National Committee (JNC 8). JAMA. 2014;311(5):507-520.

21. Chung K, Stewart I, Gisler C, et al. The Acute Kidney Injury Network (AKIN) Criteria Applied in Burns. J Burn Care Res. 2012;33(4):483-490.

22. Coca S, Bauling P, Schifftner T, Howard C, Teitelbaum I, Parikh C. Contribution of acute kidney injury toward morbidity and mortality in burns: a contemporary analysis. Am J Kidney Dis. 2007;49(4):517-523.

23. Steinvall I, Bak Z, Sjoberg F. Acute kidney injury is common, parallels organ dysfunction or failure, and carries appreciable mortality in patients with major burns: a prospective exploratory cohort study. Crit Care. 2008;12(5):R124.

24. Thurlow J, Abbott K, Linberg A, Little D, Fenderson J, Olson S. SCr and SCysC concentrations before and after traumatic amputation in male soldiers: a casecontrol study. Am J Kidney Dis. 2014;63(1):167-170.

25. Im E, Stewart I, Morrow B, et al. Retrospective review of serum creatinine and creatinine-based measures of estimated glomerular filtration rate in an amputee population. Mil Med. 2012;177(8):952-956.

26. Stewart I, Faulk T, Sosnov J, et al. Rhabdomyolysis among critically ill combat casualties: Associations with acute kidney injury and mortality. J Trauma Acute Care Surg. 2016;80(3):492-498.

27. Kellum J, Sileanu F, Murugan R, Lucko N, Shaw A, Clermont G. Classifying AKI by Urine Output versus Serum Creatinine Level. J Am Soc Nephrol. 2015;26(9):2231-2238.

28. Siew E, Peterson J, Eden S, Moons K, Ikizler T, Matheny M. Use of multiple imputation method to improve estimation of missing baseline serum creatinine in acute kidney injury research. Clin J Am Soc Nephrol. 2013;8(1):10-18. 
Table 1. Baseline characteristics of patient cohort

\begin{tabular}{|l|c|c|c|c|}
\hline & $\begin{array}{c}\text { Full } \\
\text { Cohort } \\
(\mathbf{N = 3 8 0 7})\end{array}$ & $\begin{array}{c}\text { AKI } \\
(\mathbf{n = 4 7 4})\end{array}$ & $\begin{array}{c}\text { No AKI } \\
(\mathbf{n = 3 3 3 3})\end{array}$ & P value \\
\hline Age (y) & $26 \pm 6$ & $27 \pm 7$ & $25 \pm 6$ & 0.002 \\
\hline Male sex & 98.3 & 98.5 & 98.3 & 0.7 \\
\hline Race & & & & 0.06 \\
$\quad$ Non-African American & 64.4 & 61.2 & 64.9 & \\
$\quad$ African American & 5.4 & 7.6 & 5.1 & \\
$\quad$ Missing & 30.2 & 31.2 & 30.0 & $<0.001$ \\
\hline ISS & $16[10-24]$ & $26[17-36]$ & $14[9-22]$ & $<0.001$ \\
\hline Amputation Injury & 19.0 & 28.3 & 17.7 & $<0.001$ \\
\hline Burn Injury & 18.9 & 28.1 & 17.6 & 0.5 \\
\hline Mechanism of injury & & & & \\
Gunshot wound & 18.2 & 19.2 & 18.0 & 0.04 \\
Explosive & 79.0 & 78.1 & 79.2 & $<.8$ \\
$\quad$ Other & 2.8 & 2.7 & 2.8 & \\
\hline Heart rate, beats/min & $97 \pm 25$ & $107 \pm 28$ & $96 \pm 25$ & 0.001 \\
\hline Temperature, ${ }^{\circ} \mathrm{F}$ & $98.3 \pm 1.7$ & $98.5 \pm 2.1$ & $98.3 \pm 1.7$ & \\
\hline MAP category & 14.1 & 17.5 & 13.6 & \\
High: $>106$ mm Hg & 75.6 & 62.7 & 77.4 & \\
Normal: 65-106 mm Hg & 10.3 & 19.8 & 9.0 & \\
Low: $<65$ mm Hg & & & \\
\hline
\end{tabular}

Note: Values for categorical variables are given as percentages; values for continuous variables, as mean \pm standard deviation or median [interquartile range].

AKI, acute kidney injury; ISS, injury severity score; MAP= Mean arterial blood pressure 
Table 2. Univariate and multivariable logistic regression models for development of AKI

\begin{tabular}{|c|c|c|c|c|}
\hline \multirow[b]{3}{*}{ Age, per 1-year older } & \multicolumn{2}{|c|}{ Univariate Analysis } & \multicolumn{2}{|c|}{ Multivariable Analysis } \\
\hline & OR $(95 \% \mathrm{Cl})$ & & OR $(95 \% \mathrm{Cl})$ & \\
\hline & $1.03(1.01-1.04)$ & $<0.001$ & $1.03(1.02-1.05)$ & $<0.001$ \\
\hline Female sex & $0.86(0.39-1.90)$ & 0.7 & $0.90(0.38-2.11)$ & 0.8 \\
\hline Race & & & & \\
\hline African American & $1.57(1.07-2.29)$ & 0.02 & $1.56(1.03-2.36)$ & 0.04 \\
\hline Missing & $1.10(0.89-1.36)$ & 0.4 & $1.15(0.91-1.44)$ & 0.3 \\
\hline Non-African American & 1.00 (reference) & - & 1.00 (reference) & - \\
\hline ISS, per 1-point greater & $1.06(1.05-1.07)$ & $<0.001$ & $1.05(1.05-1.06)$ & $<0.001$ \\
\hline Amputation Injury & $1.83(1.47-2.28)$ & $<0.001$ & $1.46(1.14-1.88)$ & 0.003 \\
\hline Burn Injury & $1.83(1.47-2.28)$ & $<0.001$ & $1.77(1.37-2.27)$ & $<0.001$ \\
\hline Mechanism of injury & & & & \\
\hline Gunshot Wound & $1.08(0.85-1.38)$ & 0.5 & $1.53(1.16-2.03)$ & 0.003 \\
\hline Other & $0.99(0.55-1.78)$ & 0.9 & $1.09(0.58-2.06)$ & 0.8 \\
\hline Explosive & 1.00 (reference) & & 1.00 (reference) & \\
\hline $\begin{array}{l}\text { Heart rate, per 1-beat/min } \\
\text { greater }\end{array}$ & $1.02(1.01-1.02)$ & $<0.001$ & $1.01(1.00-1.01)$ & 0.001 \\
\hline $\begin{array}{l}\text { Temperature, per } 1^{\circ} \mathrm{F} \\
\text { greater }\end{array}$ & $1.07(1.02-1.14)$ & 0.01 & $1.08(1.05-1.84)$ & 0.01 \\
\hline MAP & & & & \\
\hline Low: <65 mm Hg & $2.73(2.10-3.54)$ & $<0.001$ & $1.60(1.20-2.14)$ & 0.002 \\
\hline High: >106 mm Hg & $1.58(1.22-2.06)$ & $<0.001$ & $1.39(1.05-1.84)$ & 0.02 \\
\hline Normal: 65-106 mm & 1.00 (reference) & - & 1.00 (reference) & 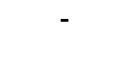 \\
\hline
\end{tabular}

Likelihood ratio: $\chi^{2}=359.05$ ( $\left.p<0.001\right)$; Hosmer-Lemeshow goodness-of-fit test: $\chi^{2}=12.79$ $(\mathrm{p}=0.1)$.

$\mathrm{OR}=$ Odds ratio

$\mathrm{CI}=$ Confidence interval

ISS = Injury severity score

$\mathrm{MAP}=$ Mean arterial blood pressure. 
Table 3. Univariate and multivariable logistic regression models for 90 day mortality

\begin{tabular}{|c|c|c|c|c|}
\hline \multirow[b]{3}{*}{ Age, per 1-y older } & \multicolumn{2}{|c|}{ Univariate Analysis } & \multicolumn{2}{|c|}{ Multivariable Analysis } \\
\hline & OR $(95 \% \mathrm{Cl})$ & $P$ value & OR $(95 \% \mathrm{Cl})$ & \\
\hline & $1.01(0.98-1.04)$ & 0.6 & $1.01(0.97-1.04)$ & 0.8 \\
\hline Female sex & $1.07(0.26-4.41)$ & 0.9 & $1.07(0.22-5.32)$ & 0.9 \\
\hline \multicolumn{5}{|l|}{ Race } \\
\hline African American & $1.68(0.85-3.30)$ & 0.1 & $1.08(0.47-2.50)$ & 0.9 \\
\hline Missing & $0.89(0.58-1.37)$ & 0.6 & $0.88(0.53-1.45)$ & 0.6 \\
\hline Non-African American & 1.00 (reference) & - & 1.00 (reference) & \\
\hline ISS, per 1-point greater & $1.08(1.07-1.09)$ & $<0.001$ & $1.07(1.06-1.08)$ & $<0.001$ \\
\hline Amputation Injury & $1.30(0.83-2.03)$ & 0.3 & $0.81(0.45-1.44)$ & 0.5 \\
\hline Burn injury & $2.01(1.33-3.02)$ & $<0.001$ & $1.50(0.88-2.57)$ & 0.1 \\
\hline \multicolumn{5}{|l|}{ Mechanism of injury } \\
\hline Gunshot Wound & $1.09(0.68-1.76)$ & 0.7 & $1.27(0.71-2.26)$ & 0.4 \\
\hline Other & $0.63(0.15-2.60)$ & 0.5 & $1.11(0.26-4.80)$ & 0.9 \\
\hline Explosive & 1.00 (reference) & - & 1.00 (reference) & - \\
\hline $\begin{array}{l}\text { Heart rate, per } 1- \\
\text { beat/min greater }\end{array}$ & $1.01(1.00-1.02)$ & 0.02 & $0.99(0.98-1.00)$ & 0.03 \\
\hline $\begin{array}{l}\text { Temperature, per } 1^{\circ} \mathrm{F} \\
\text { greater }\end{array}$ & $0.85(0.78-0.94)$ & 0.001 & $0.90(0.82-0.99)$ & 0.04 \\
\hline \multicolumn{5}{|l|}{ MAP } \\
\hline Low: <65 mm Hg & $4.62(2.96-7.20)$ & $<0.001$ & $2.33(1.36-4.01)$ & 0.002 \\
\hline High: >106 mm Hg & $2.25(1.37-3.69)$ & 0.001 & $1.98(1.13-3.47)$ & 0.02 \\
\hline Normal: $65-106 \mathrm{~mm}$ & 1.00 (reference) & & 1.00 (reference) & \\
\hline \multicolumn{5}{|l|}{$\mathrm{Hg}$} \\
\hline \multicolumn{5}{|l|}{ AKI } \\
\hline KDIGO 1 & $4.91(3.02-7.98)$ & $<0.001$ & $2.88(1.69-4.92)$ & $<0.001$ \\
\hline KDIGO 2 & $20.43(10.54-39.59)$ & $<0.001$ & $6.76(3.22-14.18)$ & $<0.001$ \\
\hline KDIGO 3 & $76.03(38.73-149.23)$ & $<0.001$ & $39.36(17.61-88.02)$ & $<0.001$ \\
\hline No AKI & 1.00 (reference) & 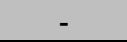 & 1.00 (reference) & - \\
\hline
\end{tabular}

Note: Likelihood ratio: $\chi 2=316.05$ ( $\mathrm{p}<0.001)$; Hosmer-Lemeshow goodness-of-fit test:

$\chi 2=9.05(\mathrm{p}=0.3)$.

$\mathrm{OR}=$ Odds ratio

$\mathrm{CI}=$ Confidence interval

ISS= Injury severity score

$\mathrm{MAP}=$ Mean arterial blood pressure $\mathrm{AKI}=$ Acute kidney injury

KDIGO= Kidney Disease: Improving Global Outcomes

Odds ratios represent per one unit increase in age, ISS, heart rate and temperature 
Figure 1. Mortality stratified by Kidney Disease Improving Global Outcomes (KDIGO) acute kidney injury stage. P value for the trend across groups.

Figure 2. Hospital day of diagnosis in patients that developed acute kidney injury. 


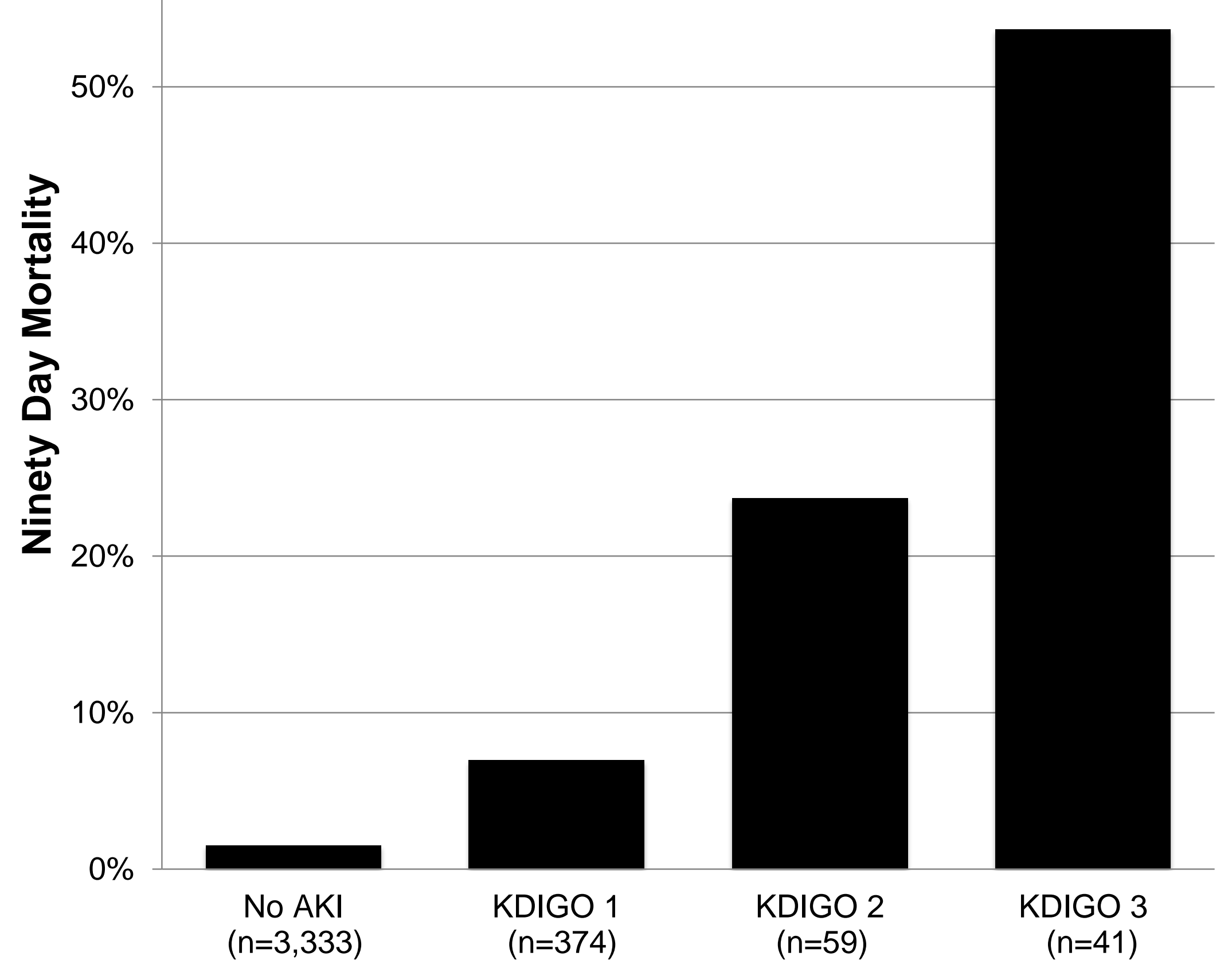




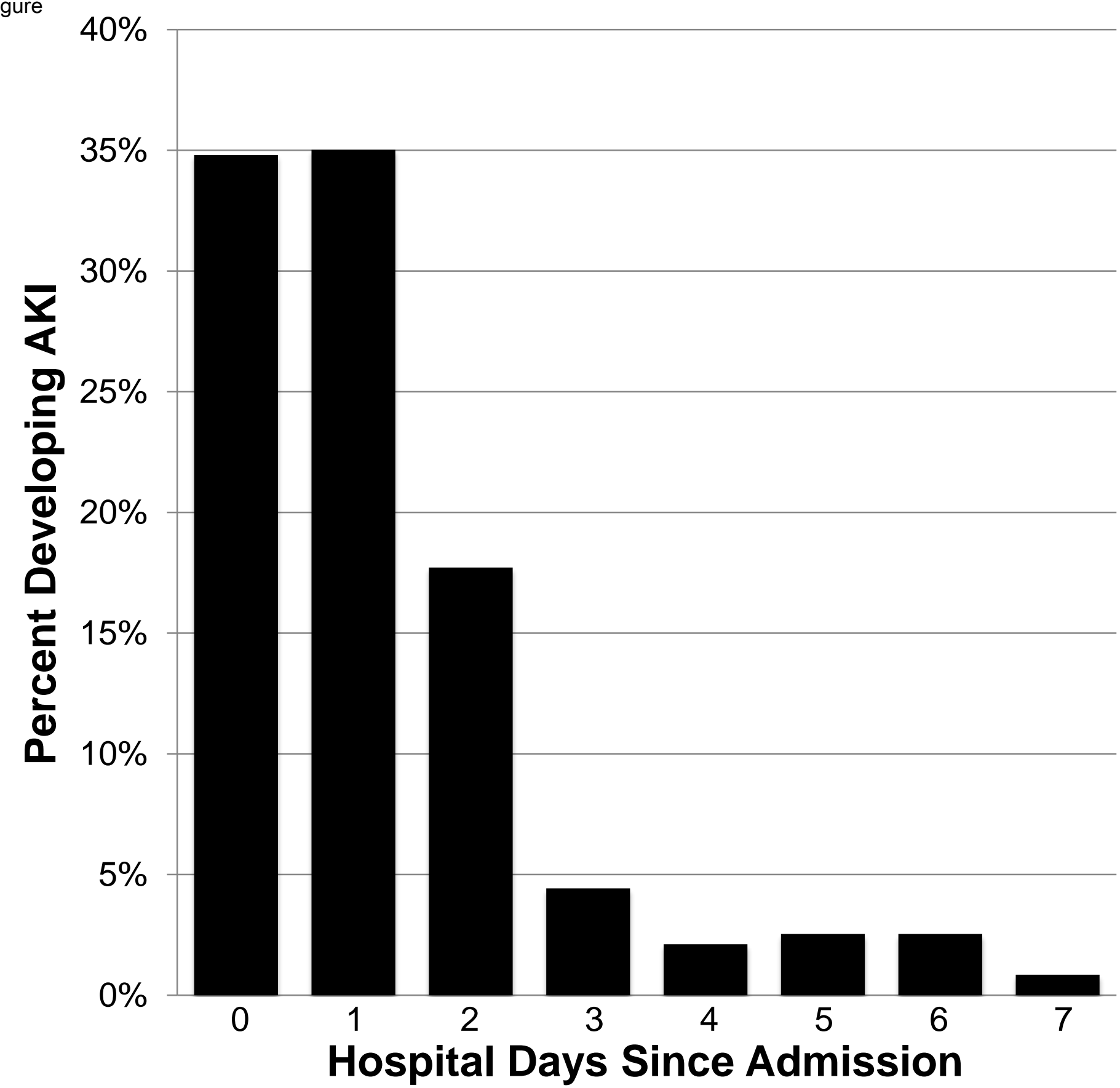

Hospital Days Since Admission 